\title{
THERMAL DISPERSION AND MELTING IMPACTS ON MIXED CONVECTIVE NON-NEWTONIAN FLUID FLOW
}

\author{
J. SIVA RAM PRASAD*, D. RAJANI, M. V. D. N. S. MADHAVI \& K. HEMALATHA
}

V R Siddhartha Engineering College, Vijayawada, Andhra Pradesh, India

\begin{abstract}
This paper targets analyzing the impact of melting and heat dispersion on non-Darcy mixed convection transfer of heat flow past a vertical front implanted in a pores structured region filled with non-Newtonian liquid. The conditions governing the stream are comprehended numerically by utilizing the Runge-Kutta strategy of $4^{\text {th }}$ order under the scheme of shooting system.
\end{abstract}

KEYWORDS: Melting, Mixed Convection, Dispersion \& Pores Structured Region

Received: Jun 06, 2020; Accepted: Jun 26, 2020; Published: Jul 01 2020; Paper Id.: IJMPERDJUN2020167

\section{INTRODUCTION}

In numerous procedures when related with stage change, transfer of heat happens with melting. Investigations of this problem have wide variety of applications, for example, in throwing, welding and magma cementing. The liquefying of permafrost, the defrosting of solidified grounds and planning of semiconducting materials are a few zones that include transfer of heat with melting. In the investigations of transfer of heat when related with liquification in a permeable region Kazmierczaketal.[1] analyzed the flow rate, temperature and Nusselt number from a level plate in the melting zone within the sight of relentless common convection. Bakier[2] considered the dissolving impact on blended convection along vertical plate of arbitrary wall temperature in supporting and contradicting streams in a liquid immersed permeable region. It was seen that at solid fluid interface, the process of melting declines the relative Nusselt number.

Gorla et al.[3] concentrated on the issue of convection of mixed in dissolving from a vertical geometry of stable temperature in a soaked permeable region. It was seen that the melting phenomena is practically equivalent to mass infusion and blowing close to the limit and along these lines decreases the transfer of heat through solid fluid interface.

Further, non-Newtonian power law liquids are especially wide spread in modern procedure and in nature. The dissolving marvels on convection along a vertical front in a non-Newtonian liquid immersed permeable network are examined by Poulikakos and Spatz [4].Nakayama and Shenoy [5] examined a bound together closeness change for non-Darcy and Darcy constrained, natural and blended convective heat exchange in nonNewtonian inelastic liquid immersed permeable region by considering the oil repository designing and geothermal applications

The impact of thermo-dissemination and melting on characteristic convective heat mass exchange in a soaked non-Darcy permeable structure with non-Newtonian liquid was considered by Kairi and Murthy [6]. The non-straight conduct of non-Newtonian liquids in a permeable framework is very not quite the same as that of 
Newtonian liquids in permeable media. Melting and radiation consequences for blended convection past a vertical surface inserted in a non-Newtonian liquid immersed non-Darcy permeable mechanism for aiding and restricting outer streams was examined by Ali J. Chamkaet al.[7].

The investigation of thermal scattering impacts becomes common in the permeable media stream zone. Hong and Tien [8] analyzed diagnostically the impact of transverse heat scattering on characteristic convection from a vertical, warmed plate in a permeable region. Their outcomes demonstrate that because of the better blending of the thermal scattering impact, the rate of heat transport is expanded. Hemalatha and JSR Prasad [9] contemplated impact of radiation on liquification from a vertical plate installed in permeable region in MHD blended convection with viscous dispersal under the conditions of non-Darcy (Forchheimer). Mahantheshet al.[10] examined the highlights of heat and mass exchange impacts in non-Newtonian Casson liquid towards a precarious penetrable extending surface. Rajaniet al.[11] dissected the transfer of heat qualities past a vertical plate with variable temperature inserted in a non-Darcy permeable medium.

The current article analyses the force of heat dispersion with melting phenomenon on convective heat transport in a non-Darcy pores structured region pasta vertical geometrical surface.

\section{MATHEMATICAL FORMULATION}

Consider the non-Newtonian mixed convective stream in a non-Darcy pores structured region through a vertical level geometry as appeared in figure 1. The dimensional directions ( $\mathrm{x}, \mathrm{y})$ are picked with the end goal that ' $\mathrm{x}$ ' is estimated in the plate direction and ' $y$ ' is orthogonal to it. The imagined plate acts as a juncture between solid fluid stages during dissolving in the midst of the permeable structure and it is at liquefying temperature $T_{m}$. The temperature of solid surface is at $\mathrm{T}_{0}$ which is less than $\mathrm{T}_{\mathrm{m}}$. A dainty layer of boundary develops near the privilege of plate and the temperature transition easily along this layer from $T_{m}$ to $T_{\infty}$, which is the encompassing liquid stage temperature away from the inter face.

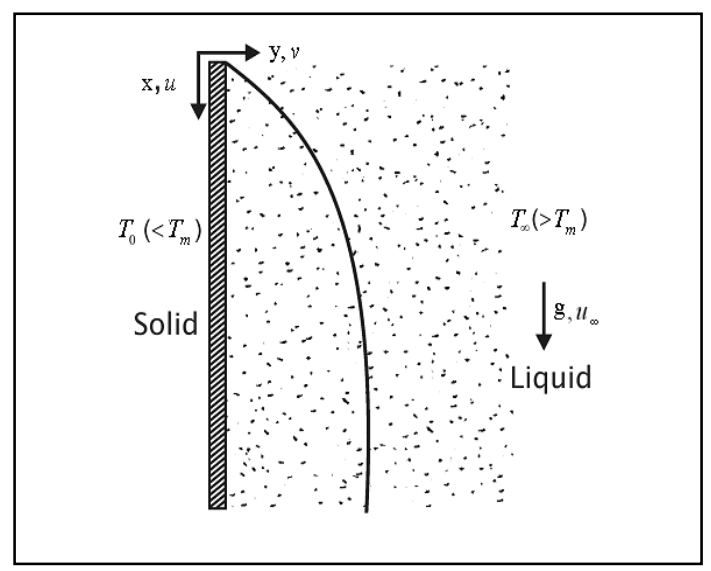

Figure 1: Schematic Sketch.

By considering the impact of thermal dispersion, the equations governing the flow are designed as:

$$
\begin{aligned}
& \frac{\partial u}{\partial x}+\frac{\partial v}{\partial y}=0 \\
& \frac{\partial u^{n}}{\partial y}+\mathrm{CK}^{1 / 2} v^{-1} \frac{\partial u^{2}}{\partial y}=\mp \frac{K g p}{v} \frac{\partial T}{\partial y}
\end{aligned}
$$


$\mathrm{u} \frac{\partial \mathrm{T}}{\partial \mathrm{x}}+\mathrm{v} \frac{\partial \mathrm{T}}{\partial \mathrm{y}}=\frac{\partial}{\partial y}\left(\alpha \frac{\partial \mathrm{T}}{\partial \mathrm{y}}\right)$ with $\alpha=\alpha_{\mathrm{m}}+\alpha_{\mathrm{d}}$

The factor in theafore mentioned equations which signifying the buoyancy forced impact in the flow region has Findications. The negative notation denotes the aiding buoyancy flow whereas the positive notation signifies buoyancy opposed flow. Here, $\mathrm{u}$ and vare components of flow rate along dimensional coordinates (x, y), $\mathrm{C}$ - Empirical constant of Forchheimer, $\mathrm{n}$ - Power-law fluid viscosity index, T- Heat boundary-layer temperature, $\mathrm{K}$ - Permeability, $\beta$ - Coefficient of heat expansion, $v$ - Kinematics viscosity, $g$-Gravitational acceleration, $\alpha$ Thermal diffusivity and $\alpha_{\mathrm{m}}$ - Molecular diffusivity. As per the model initiated by Plumb [12], the dispersion thermal diffusivity $\alpha_{\mathrm{d}}$ is directly related to the component of velocity i.e $\alpha_{\mathrm{d}}=\gamma \mathrm{ud}$, where $\gamma$ - Coefficient of dispersion and d - Mean particle diameter.

The associated conditions at boundary:

$$
\begin{aligned}
& \mathrm{y}=0, \mathrm{~T}=\mathrm{T}_{\mathrm{m}}, \rho \mathrm{v}\left[\mathrm{h}_{\mathrm{sf}}+\left(\mathrm{T}_{\mathrm{m}}-\mathrm{T}_{0}\right) \mathrm{c}_{\mathrm{s}}\right]=\mathrm{k} \frac{\partial \mathrm{T}}{\partial \mathrm{y}} \\
& \mathrm{y} \rightarrow \infty, \mathrm{u} \rightarrow \mathrm{u}_{m b}, \mathrm{~T} \rightarrow \mathrm{T}_{\infty}
\end{aligned}
$$

Where $\mathrm{h}_{\mathrm{sf}}$ - Solid latent heat, $\mathrm{c}_{\mathrm{g}}$ - Solid phase specific heat, and $\mathrm{u}_{\mathrm{sg}}$ - Aiding external flow velocity, $\mathrm{k}(=$ $\rho \alpha c_{p}$ )- Permeable region effective thermal conductivity. The continuity equation (1) will be fulfilled by induction of stream function $\psi$ for incompressible flow as $u=\Psi_{y}$ and $v=-\Psi_{x}$. Now the equations (2-3) are altered into

$$
\begin{aligned}
& \frac{\partial}{\partial y}\left(\frac{\partial v}{\partial y}\right)^{\mathrm{n}}+\mathrm{CK}^{1 / 2} \mathrm{v}^{-1} \frac{\partial}{\partial y}\left(\frac{\partial v}{\partial y}\right)^{2}=\mp \frac{\mathrm{Kg} p}{v} \frac{\partial \mathrm{T}}{\partial y} \\
& \frac{\partial \psi}{\partial y}\left(\frac{\partial \mathrm{T}}{\partial \mathrm{x}}\right)-\frac{\partial \psi}{\partial \mathrm{x}}\left(\frac{\partial \mathrm{T}}{\partial y}\right)=\frac{\partial}{\partial y}\left[\left(\alpha_{\mathrm{m}}+(\gamma) \frac{\partial v}{\partial y} \mathrm{~d}\right) \frac{\partial \mathrm{T}}{\partial y}\right]
\end{aligned}
$$

Introduce the similarity variable $(\eta)$ as $\psi=f(\eta)\left(u_{m x} \alpha_{m} x\right)^{0.5}, \eta=\left(\frac{x u_{m}}{a_{m}}\right)^{0.5}\left(\frac{y}{x}\right), \theta(\eta)=\frac{T-T_{m}}{T_{x x}-T_{m}}$.

Now the equations (4-5) together with (6-7) are transformed as

$$
\begin{aligned}
& \mathrm{nf}^{11} \mathrm{f}^{1^{\mathrm{n}-1}}+2 \mathrm{Ff}^{1} \mathrm{f}^{11}=\mp\left(\frac{\mathrm{Ra}_{\mathrm{x}}}{\mathrm{Pe}_{\mathrm{x}}}\right)^{\mathrm{n}} \theta^{1} \\
& \left(1+\mathrm{Df}^{1}\right) \theta^{11}+\left(\frac{1}{2} \mathrm{f}+\mathrm{Df}^{11}\right) \theta^{1}=0 \\
& \eta=0, \theta=0,\left\{\mathrm{Df}^{1}(0)+1\right\} 2 \mathrm{M}^{1}(0)+\mathrm{f}(0)=0 . \\
& \eta \rightarrow \infty, \theta=\mathrm{f}^{1}=1 .
\end{aligned}
$$

Here the representation of prime is the derivative corresponding to $\eta$. The dimensionless parameters used

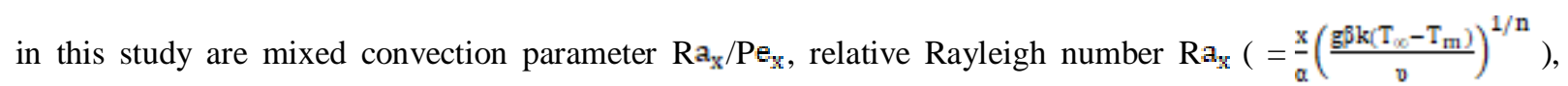
relative Peclet number $\mathrm{Pe}_{\mathrm{S}}\left(=\frac{\mathrm{u}_{\mathrm{g} x} \mathrm{x}}{\mathrm{a}}\right)$, non-Darcian parameter $\mathrm{F}\left(=\mathrm{f}_{0}\left(\mathrm{Pe}_{\mathrm{d}}\right)^{2-\mathrm{n}, \mathrm{f}_{0}}=\left(\frac{\mathrm{a}}{\mathrm{d}}\right)^{\mathrm{z}-\mathrm{n}}\left(\frac{\mathrm{C} \sqrt{\mathrm{K}}}{\mathrm{v}}\right)\right)$, pore diameter

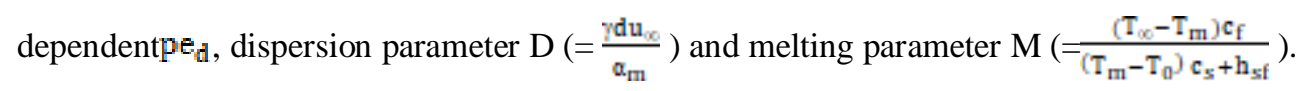

The applied-flux $\mathrm{q}_{\mathrm{w}}$ at the surface of plate $(\mathrm{y}=0)$ is $-\mathrm{k}\left(\frac{\partial \mathrm{T}}{\partial \mathrm{y}}\right)$. 
The relative Nusselt number is $\mathrm{N}_{\mathrm{ul}}=\frac{\mathrm{hx}}{\mathrm{k}}=\frac{x q_{\mathrm{w}}}{\mathrm{k}\left(\mathrm{T}_{\mathrm{m}}-\mathrm{T}_{\mathrm{m}}\right)}$, where $\mathrm{h}-$ Relative coefficient of heat flow and $\mathrm{k}-$ Effective thermal conductivity of pore structured region. In dimensionless transformed form, the Nusselt number is

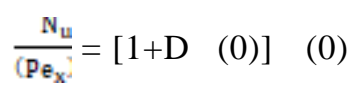

\section{SOLUTION PROCEDURE}

Equations (8-9) coupled with conditions (10-11) are tackled numerically by implementing R-K method of $4^{\text {th }}$ order coupled with reliable shooting scheme. The final solution attained is correlated for the values of $\mathrm{f}^{\mathbb{1}}(\infty)$ and $\theta$ (0). At boundary, $\eta \rightarrow \infty$ is rounded by $\rightarrow \rightarrow 8$ foranalyzing the profiles of flow rate and temperature to proceed towards the pertinent properties of free stream.

\section{RESULTS AND DISCUSSIONS}

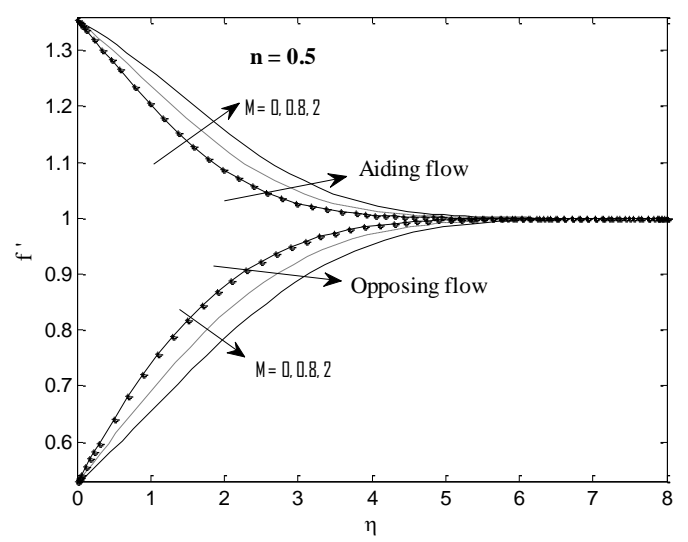

Figure 2: Impact of Melting on Velocity Component.

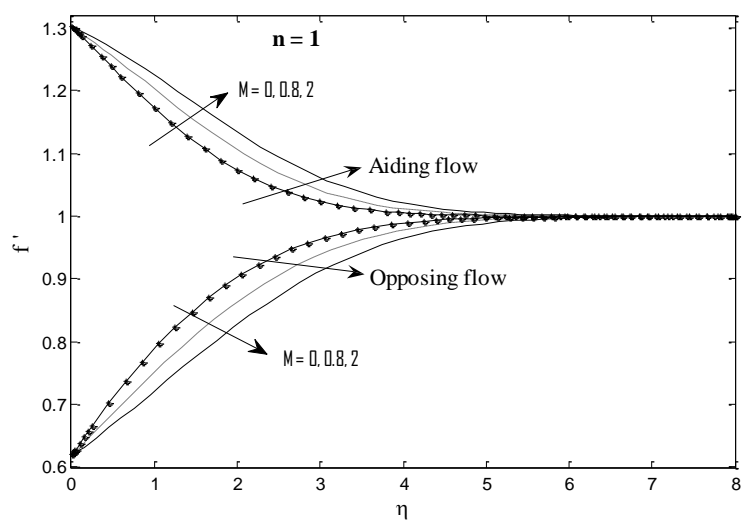

Figure 3: Impact of Melting on Velocity Component. 


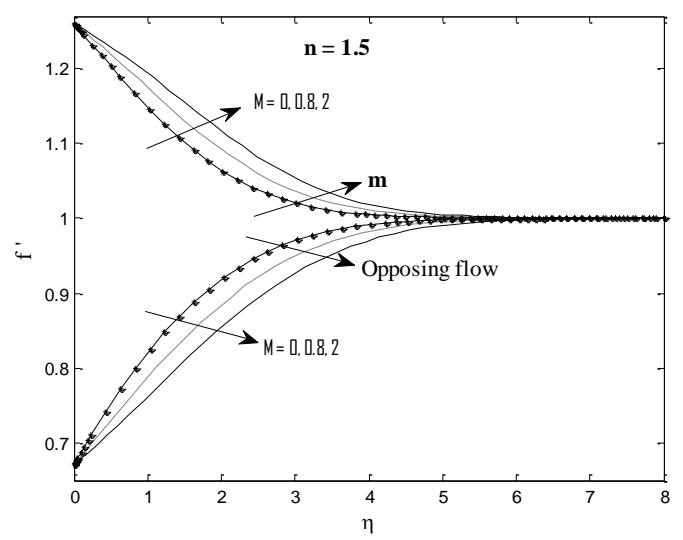

Figure 4: Impact of Melting on Velocity Component.

Figures 2 to 4 showcase the influential behavior of fluid velocity across the boundary-layer with melting parameter $\mathrm{M}$ for pseudo-plastic, Newtonian and dilatant fluids respectively, with the inertial coefficient $\mathrm{F}=1$, $\mathrm{D}=0.5$ and $\frac{\mathrm{Ra}}{\mathrm{Pe}}=1$ in both the flow cases of opposing and aiding. The augmentation in the melting phenomenon results the enhancement in velocity profile for the situation of aiding. Also, the increase in boundary-layer thickness due to melting is noticed in figures. The decrement trend is noticed in velocity profile for the case of opposing.

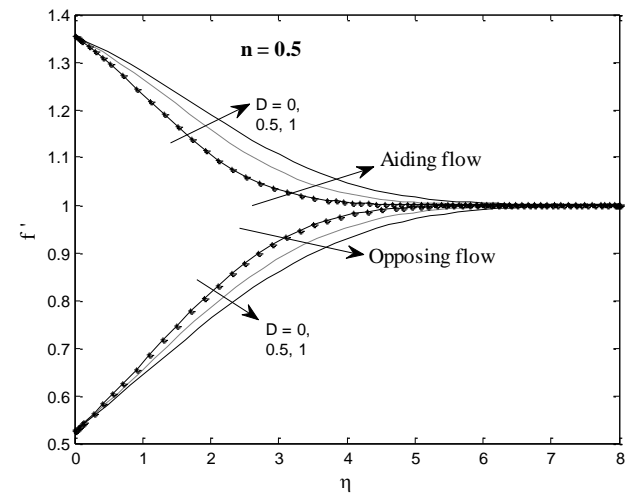

Figure 5: Thermal Dispersion Impact on Velocity Profile.

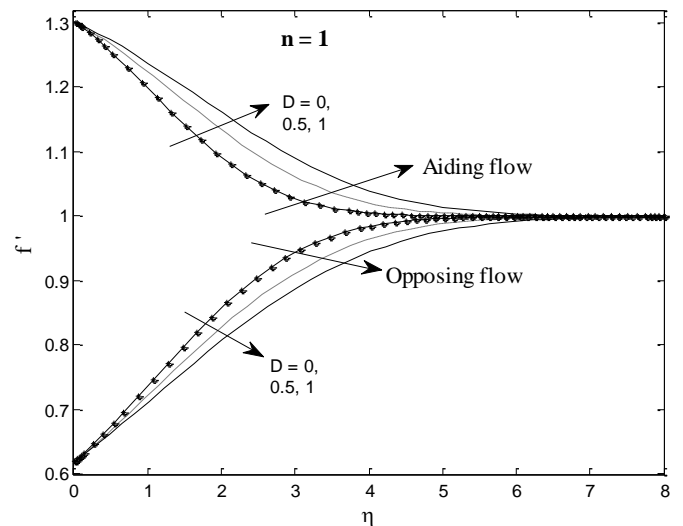

Figure 6: Thermal Dispersion Impact on Velocity Profile. 


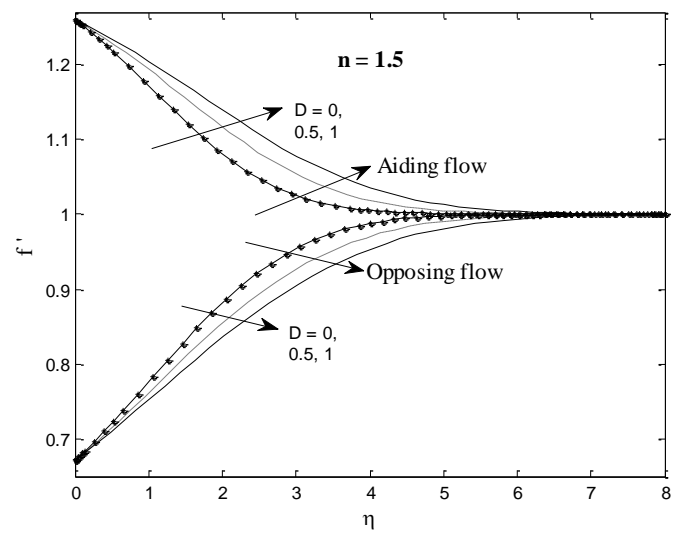

Figure 7: Thermal Dispersion Impact on Velocity Profile.

Figures 5 to 7 depict the variation of dimensionless velocity in non-Darcy regime with distinct parametric values of the dispersion $\mathrm{D}$ in both the situations of flow for pseudo-plastic, Newtonian and dilatant fluids respectively with the fixed inertial coefficient $F=1, \quad=1$ and $M=2$. The enhancement in velocity profiles noticed with the surge in dispersion D with melting. Switch conduct is seen just in the flow situation of opposing.

The impact of parameters of melting $\mathrm{M}$ and dispersion $\mathrm{D}$ on variation in rate of heat flow with $\mathrm{Ra} / \mathrm{Pe}=1$, $\mathrm{F}=1$ and $\mathrm{n}=0.5$ is illustrated in Figures 8 and 9in both the flow situations assisting and opposing respectively. In both flow situations, the rate of heat flow diminishes more significantly with the augmentation in melting phenomena.But reverse conduct is noticed with the augmented values of dispersion. The influence of thermal dispersion in aiding flow is more effective on heat flow rate than when compared with opposing flow.

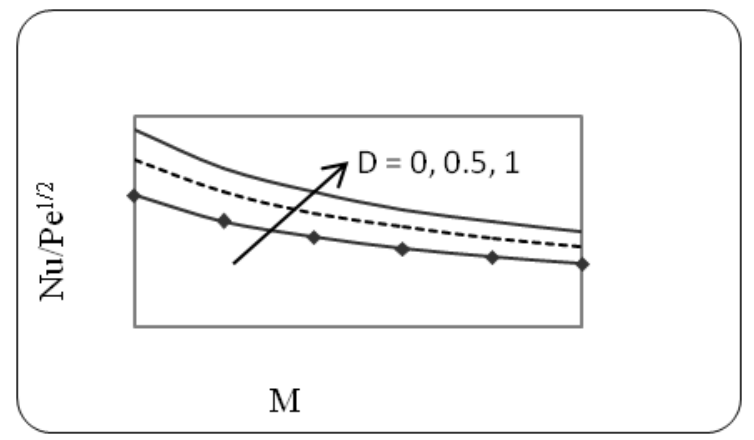

Figure 8: Heat Transfer Vs. Thermal Dispersion (Aiding Flow).

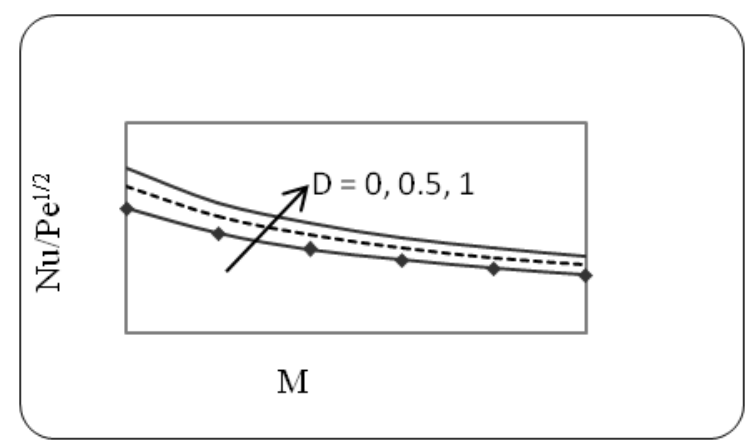

Figure 9: Heat Transfer Vs. Thermal Dispersion (Opposing Flow). 


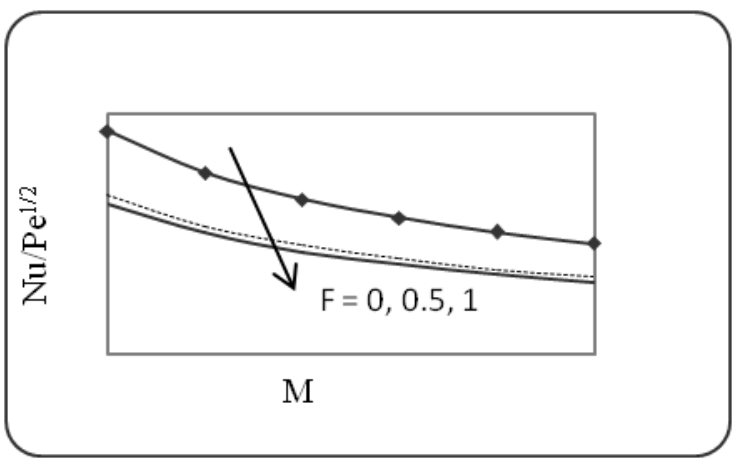

Figure 10: Heat Transfer Vs. non-Darcy Parameter with $D=0($ Aiding Flow).

Figures 10 and 11 render the force of melting $\mathrm{M}$ and inertia on heat flow rate without and with thermal dispersion $\mathrm{D}$ for aiding flow respectively with $\mathrm{Ra} / \mathrm{Pe}=1$ and $\mathrm{n}=0.5$. In non-Darcy region, the rate of heat flow diminishes with the increasing function of melting for the flow situation of aiding with the augmenting values of non-Darcy parameter F. In the presence of dispersion, the decrement in the heat flow rate is more than in absence.

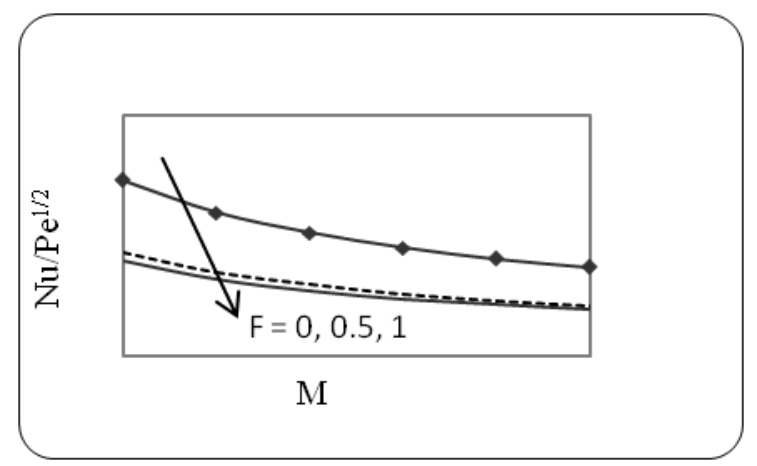

Figure 11: Heat Transfer Vs. Non-Darcy Parameter with D=0.5 (Aiding Flow).

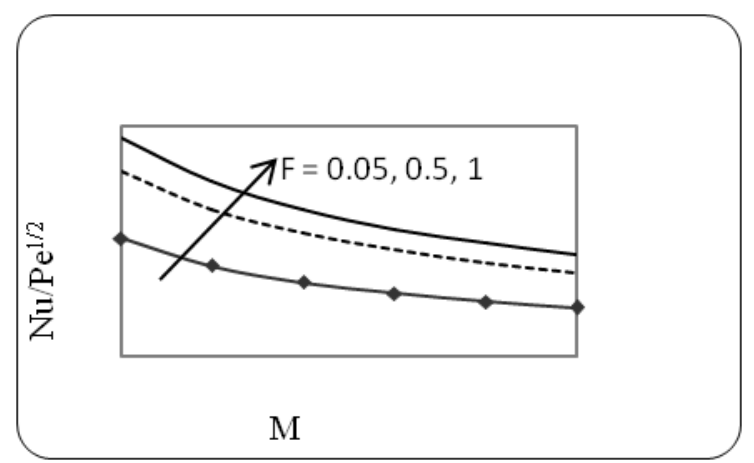

Figure 12: Heat Transfer Vs. Non-Darcy Parameter with $D=0$ (Opposing flow). 


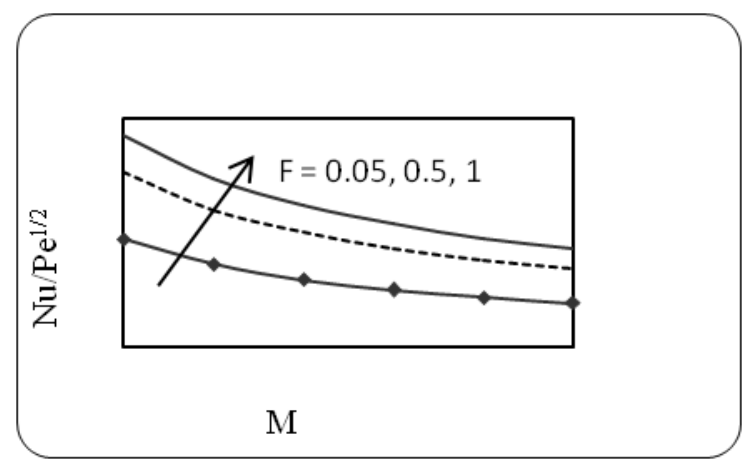

Figure 13: Heat Transfer Vs. Non-Darcy Parameter with $D=0.5$ (Opposing Flow).

Figures 12 and 13 depict the impact of melting phenomena $\mathrm{M}$ and inertia on heat flow rate without and with thermal dispersion for the case of opposing flow for fixed $\mathrm{Ra} / \mathrm{Pe}=1$ and $\mathrm{n}=0.5$.In this case, the heat flow rate is enhancing as the augmentation in the inertia parameter.

Figures 14 and 15 show the impact of viscosity index parameter $\mathrm{n}$ of fluid and melting $\mathrm{M}$ on the rate of heat flow with dispersion parameter $\mathrm{D}(=0.5)$ and inertial coefficient $\mathrm{F}(=1)$ for aiding and opposing fluid flows respectively. It is observed that the heat flow rate falls with the enhancement in the viscosity index value in assisting flow. But in the flow of opposing, the rate of heat flow rises with the enhancement in fluid viscosity index $\mathrm{n}$. The viscosity index of the fluid is more effective on heat transfer rate in opposing flow than when compared with aiding case.

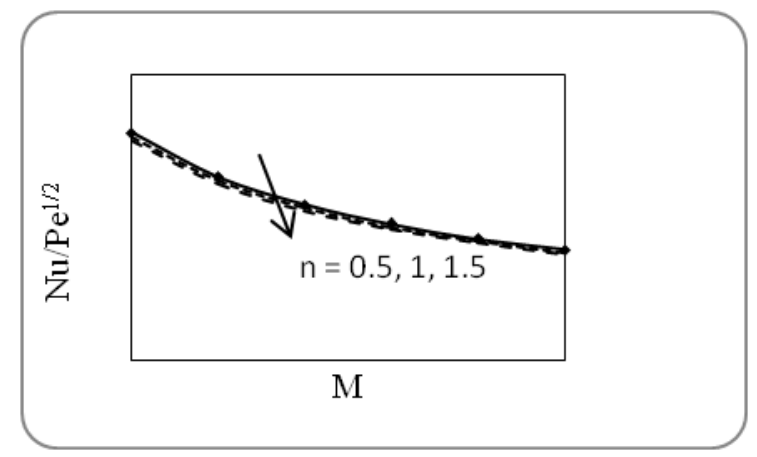

Figure 14: Nusselt Number Vs. Fluid Viscosity Index (Aiding Flow).

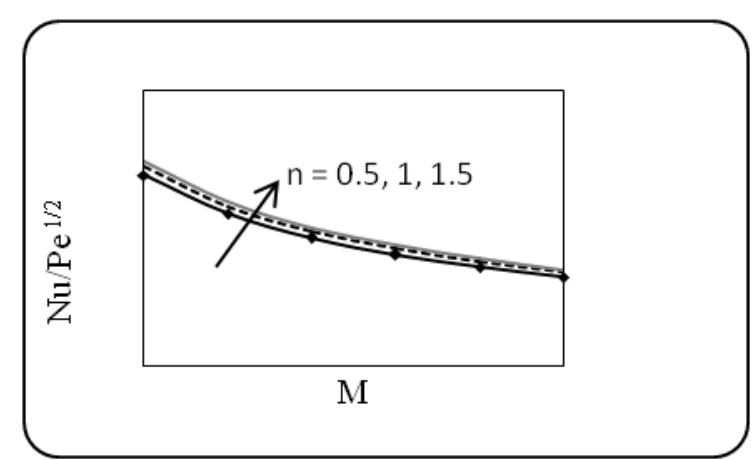

Figure 15: Nusselt Number Vs. Fluid Viscosity Index (Opposing Flow). 


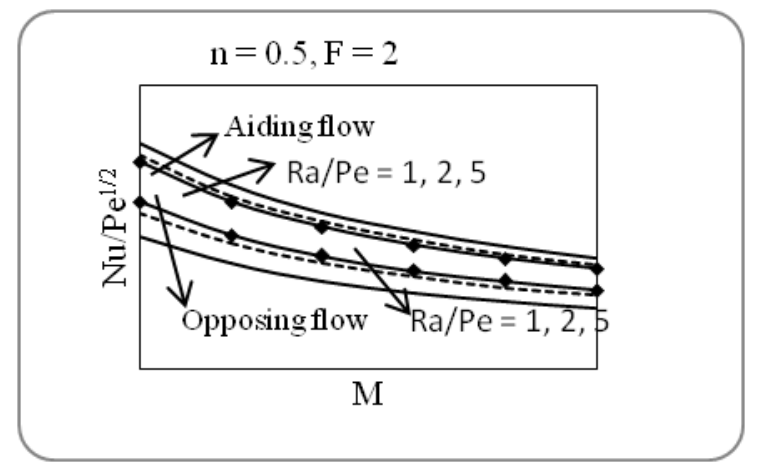

Figure 16: Nusselt Number Vs. Mixed Convection Parameter.

The analysis of mixed convectionvia melting in the presence of dispersion $\mathrm{D}(=1)$ on heat transport is presented in Figure 16 for both the flows (aiding and opposing). It is noticed that the heat flow rate falls prominently with the surge in melting strength M. Further it is notified that in aiding flow, as mixed convection parameter rises Nusselt number increases. But in opposing flow switch nature is noticed.

\section{CONCLUSIONS}

The rise in parameter values of melting and dispersion results in an enhancement in profiles of velocity for the situation of assisting and reverse conduct are noticed for opposing situation.

The increasing dispersion parameter yields to an enhancement in Nusselt number for the both flow cases. The rate of heat flow declines with the enhancement in the fluid viscosity index values and the values of nonDarcy parameter in assisting flow. Reverse nature is noticed in the flow case of opposing. The rise of melting parameter leads to a prominent drop in heat flow rates in both the flow situations.

The rate of heat flow enhances with the augmentation in parameter of mixed convection in the situation of assisting and reverse conduct is noticed in the opposing case.

\section{REFERENCES}

1. M. Kazmierczak, D. Poulikakos and I. Pop, "Melting from a Flat Plate Embedded in a Porous Medium in the Presence of Steady Natural Convection”, Numerical Heat Transfer, vol. 10, issue. 6, (1986), pp. 571-581.

2. A.Y. Bakier, "Aiding and Opposing Mixed Convection Flow in Melting from a Vertical Flat Plate Embedded in a Porous Medium”, Transport in Porous Media, vol. 29, (1997), pp.127-139.

3. Kavitha, P., and N. Kishan. "Quasilinearization approach to mhd heat transfer to non-newtonian power-law fluids flowing over a wedge with heat source/sink in the presence of viscous dissipation." Int. J. Math. Comput. Appl. Res 3 (2013): 15-28.

4. Rama Subba Reddy Gorla, M. A. Mansour, I. A. Hassanien and A. Y. Bakier, "Mixed Convection Effect on Melting from a Vertical Plate in a Porous Medium”, Transport in Porous Media, vol. 36, (1999), pp. 245-254.

5. Begam, M. Jannath, and M. Deivanayaki. "An Unsteady Pulsatile Flow of Some Mhd Non-Newtonian Nanofluids with Hall Current and Ion Slip Through a Porous Medium. "International Journal of Mechanical and Production Engineering Research and Development (IJMPERD) 9. 4, Aug 2019, 111-118

6. D. Poulikakos and T.L. Spatz, "Non-Newtonian Natural Convection at a Melting Front in a Permeable Solid Matrix”, International Communications in Heat and Mass Transfer, vol. 15, issue. 5, (1988), pp. 593-603. 
7. A. Nakayama and A.V. Shenoy, "A Unified Similarity Transformation for Darcy and Non-Darcy Forced, Free and Mixed Convection Heat Transfer in Non- Newtonian Inelastic Fluid Saturated Porous Media”, The Chemical Engineering Journal, vol. 50, issue. 1, (1992), pp. 33-45.

8. Rao, P. Srinivasa, and G. Bhanodaya Reddy. "Effect of reflux on peristaltic motion in an asymmetric channel with partial slip and different wave forms." International journal of mechanical engineering (IJME) 3 (2014): 35-48.

9. R.R. Kairi, and P.V.S.N. Murty, "Effect of Melting and Thermo-Diffusion on Natural Convection Heat Mass Transfer in a Non-Newtonian Fluid Saturated Non-Darcy Porous Medium "The Open Transport Phenomena Journal, vol. 1, (2009), pp. 7-14.

10. Ali J. Chamkha, Sameh E. Ahmed and Abdulkareem S. Aloraier "Melting and Radiation Effects on Mixed Convection from a Vertical Surface Embedded in a Non-Newtonian Fluid Saturated Non-Darcy Porous Medium for Aiding and Opposing External Flows", International Journal of the Physical Sciences, vol. 5(7), (2010), pp. 12121224.

11. Sadek, Hasanain AA, Mohammed J. Khami, and Taleb AS Obaid. "Computer Simulation of Blood Flow in Large Arteries by a Finite Element Method." International Journal of Computer Science and Engineering (IJCSE) 2.4 (2013): 171-184.

12. J.T. Hong, and C.L. Tien, "Analysis of Thermal Dispersion Effect on Vertical Plate Natural Convection in Porous Media”, International Journal of Heat and Mass Transfer, vol. 30, issue. 1,(1987), pp. 143-150.

13. K. Hemalatha and J.S.R. Prasad, "Melting with Viscous Dissipation on MHD Radiative Flow from a Vertical Plate Embedded in Non-DarcyPorous Medium”,Journal of Applied Fluid Mechanics, vol. 8(4), (2015), pp. 747-752.

14. B. Mahanthesh, S. Manjunatha, B. J. Gireesha and Ammani Kuttan Baby, "Heat and Mass Transfer Effects on nonNewtonian Fluid Flow over an Unsteady Stretching Surface with Viscous Dissipation and Thermal Radiation",JP Journal of Heat and Mass Transfer, vol. 15(2), (2018), pp. 309-330.

15. D. Rajani, J. Siva Ram Prasad and K. Hemalatha, "Radiative MHD Flow past a Vertical Melting Front with Variable Temperature 'International Journal of Innovative Technology and Exploring Engineering (IJITEE), vol. 8(6S2), (2019), pp. 42-46.

16. O.A. Plumb, "The Effect of Thermal Dispersion on Heat Transfer in Packed Bed Boundary Layers "Proceedings of $1^{\text {st }}$ ASME-JSME Thermal Engineering Joint Conference, 2, (1983), pp. 17-21.

\section{AUTHORPROFILES}

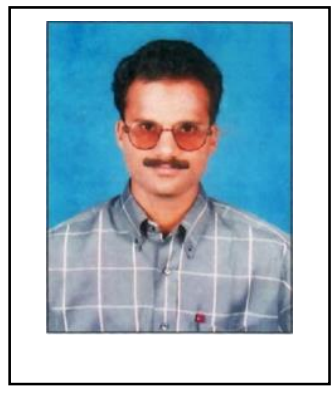

Dr. J. Siva Ram Prasad, Education details:M.Sc; B.Ed; M.Phil; Ph.D. Number of Publications: 14. Research Area: Fluid Dynamics. Membership: ISTAM. 


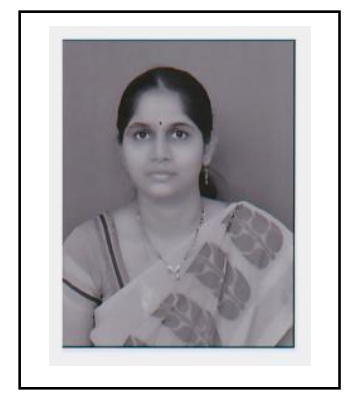

D. Rajani. Education details:M.Sc; M.Phil.Number of Publications: 7. Research Area: Fluid Dynamics.Membership: ISTAM.

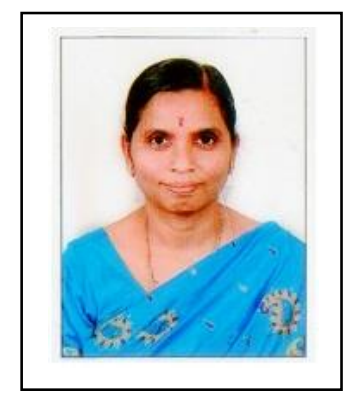

M. V. D. N. S. Madhavi. Education details:M.Sc; M.Phil. Number of Publications: 9. Research Area: Fluid Dynamics. Membership: ISTAM.

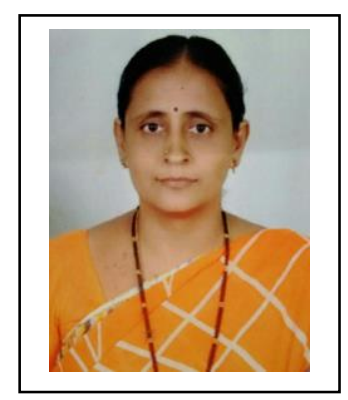

Dr. K. Hemalatha.Education details:M.Sc; M.Phil; Ph.D.Number of Publications: 34.Research work: Guided 1 Ph.D and 2 M.Tech Projects. Guiding 3 Ph.Ds and 3 M.Phils. Research Area: Fluid Dynamics. Membership: ISTAM. Achievements: Best teacher award, National meritscholarship. 
